\title{
A Class of Weak Dual Wavelet Frames for Reducing Subspaces of Sobolev Spaces
}

\author{
Jianping Zhang $\mathbb{D}$ \\ College of Mathematics and Computer Science, Yan'an University, Shaanxi 716000, China \\ Correspondence should be addressed to Jianping Zhang; zhjp198254@163.com
}

Received 21 August 2021; Accepted 6 December 2021; Published 19 January 2022

Academic Editor: Alberto Fiorenza

Copyright (c) 2022 Jianping Zhang. This is an open access article distributed under the Creative Commons Attribution License, which permits unrestricted use, distribution, and reproduction in any medium, provided the original work is properly cited.

In recent years, dual wavelet frames derived from a pair of refinable functions have been widely studied by many researchers. However, the requirement of the Bessel property of wavelet systems is always required, which is too technical and artificial. In present paper, we will relax this restriction and only require the integer translation of the wavelet functions (or refinable functions) to form Bessel sequences. For this purpose, we introduce the notion of weak dual wavelet frames. And for generality, we work under the setting of reducing subspaces of Sobolev spaces, we characterize a pair of weak dual wavelet frames, and by using this characterization, we obtain a mixed oblique extension principle for such weak dual wavelet frames.

\section{Introduction}

Let $\mathscr{H}$ be a separable Hilbert space. An at most countable sequence $\left\{e_{i}\right\}_{i \in I}$ in $\mathscr{H}$ is called a frame for $\mathscr{H}$ if there exist two constants $0<C_{1} \leq C_{2}<\infty$ such that

$$
C_{1}\|f\|^{2} \leq \sum_{i \in I}\left|\left\langle f, e_{i}\right\rangle\right|^{2} \leq C_{2}\|f\|^{2} \text { for } f \in \mathscr{H}
$$

where $C_{1}$ and $C_{2}$ are called lower and upper frame bounds; it is called a Bessel sequence in $\mathscr{H}$ if the right-hand side inequality in (1) holds, where $C_{2}$ is called a Bessel bound. Given a frame $\left\{e_{i}\right\}_{i \in I}$ for $\mathscr{H}$, a sequence $\left\{\tilde{e}_{i}\right\}_{i \in I}$ is called a dual of $\left\{e_{i}\right\}_{i \in I}$ if it is a frame such that

$$
f=\sum_{i \in I}\left\langle f, \tilde{e}_{i}\right\rangle e_{i} \text { for } f \in H .
$$

It is well-known that $\left\{e_{i}\right\}_{i \in I}$ is also a dual of $\left\{\tilde{e}_{i}\right\}_{i \in I}$ if $\left\{\tilde{e}_{i}\right\}_{i \in I}$ is a dual of $\left\{e_{i}\right\}_{i \in I}$. So, in this case, we also say ( $\left.\left\{e_{i}\right\}_{i \in I},\left\{\tilde{e}_{i}\right\}_{i \in I}\right)$ is a pair of dual frames. The fundamentals of frames can be found in [1-3]. We denote by $\mathbb{Z}, \mathbb{N}$, and $\mathbb{N}_{0}$ the set of integers, the set of positive integers, and the set of nonnegative integers, respectively. Let $d \in \mathbb{N}$ and $M$ a $d \times d$ expansive matrix (an integer matrix with all its eigen- values being greater than 1 in modulus). The dilation operator $D$ and the shift operator $T_{k}$ with $k \in \mathbb{Z}^{d}$ are, respectively, defined by

$$
\begin{gathered}
D f(\cdot)=|\operatorname{det} M|^{1 / 2} f(M \cdot), \\
T_{k} f(\cdot)=f(\cdot-k),
\end{gathered}
$$

for $f \in L^{2}\left(\mathbb{R}^{d}\right)$, and naturally extended to tempered distribution. In what follows, we denote by $M^{*}$ the transpose of $M$, and by

$$
\Gamma_{M^{*}}=\left\{\varepsilon_{0}, \varepsilon_{1}, \cdots, \varepsilon_{|\operatorname{det} M|-1}\right\}
$$

a set of representatives of distinct cosets of $\mathbb{Z}^{d} / M^{*} \mathbb{Z}^{d}$ with $\varepsilon_{0}=0$.

The Fourier transform of a function $f \in L^{1}\left(\mathbb{R}^{d}\right) \cap L^{2}\left(\mathbb{R}^{d}\right)$ is defined by

$$
\widehat{f}(\cdot)=\int_{\mathbb{R}^{d}} f(x) e^{-2 \pi i\langle x,\rangle} d x
$$

and naturally extended to tempered distribution, where $\langle\cdot$, .) denotes the Euclidean inner product in $\mathbb{R}^{d}$. Similarly, 
the inverse Fourier transform of a function $f \in L^{1}\left(\mathbb{R}^{d}\right) \cap L^{2}$ $\left(\mathbb{R}^{d}\right)$ is defined by

$$
\breve{f}(\cdot)=\int_{\mathbb{R}^{d}} f(x) e^{2 \pi i\langle x,\rangle} d x
$$

and extended to tempered distribution as usual. For a real number $s$, we denote by $H^{s}\left(\mathbb{R}^{d}\right)$ the Sobolev space consisting of all distributions $f$ such that

$$
\|f\|_{H^{s}\left(\mathbb{R}^{d}\right)}^{2}=\int_{\mathbb{R}^{d}}|f \wedge(\xi)|^{2}\left(1+|\xi|^{2}\right)^{s} d \xi<\infty,
$$

where $|\xi|$ denotes its Euclidean norm for $\xi \in R^{d}$. It is easy to check that $H^{s}\left(\mathbb{R}^{d}\right)$ is a Hilbert space under the inner product

$$
\langle f, g\rangle_{H^{s}\left(\mathbb{R}^{d}\right)}=\int_{\mathbb{R}^{d}} \widehat{\hat{f}}(\xi) \hat{\mathcal{g}}(\xi)\left(1+|\xi|^{2}\right)^{s} d \xi \text { for } f, g \in H^{s}\left(\mathbb{R}^{d}\right) .
$$

In particular, $H^{0}\left(\mathbb{R}^{d}\right)=L^{2}\left(\mathbb{R}^{d}\right)$ by the Plancherel theorem. For $\Omega \subset \mathbb{R}^{d}$ with nonzero measure, we write

$$
\begin{gathered}
F H^{s}(\Omega)=\left\{f \in H^{s}\left(\mathbb{R}^{d}\right): \operatorname{supp}(\widehat{f}) \subset \Omega\right\}, \\
F H^{0}(\Omega)=F L^{2}(\Omega) .
\end{gathered}
$$
$(\Omega)$,

Then, $F H^{s}\left(\mathbb{R}^{d}\right)=H^{s}\left(\mathbb{R}^{d}\right)$. Obviously, for each $g \in F H^{-s}$

$$
\langle f, g\rangle=\int_{\mathbb{R}^{d}} \widehat{\hat{f}}(\xi) \hat{\mathfrak{g}}(\xi) d \xi \text { for } f \in F H^{s}(\Omega)
$$

defines a continuous linear function on $F H^{s}(\Omega)$. Then, $(F$ $\left.H^{s}(\Omega), F H^{-s}(\Omega)\right)$ forms a pair of dual spaces; so does $\left(H^{s}(\right.$ $\left.\mathbb{R}^{d}\right), H^{-s}\left(\mathbb{R}^{d}\right)$ ) by taking $\Omega=\mathbb{R}^{d}$. Write

$$
\mathscr{D}=\left\{f: \widehat{f} \in L^{\infty}\left(\mathbb{R}^{d}\right) \text { and supp }(\widehat{f}) \text { is bounded }\right\} .
$$

It is well known that $\mathscr{D}$ is dense in $H^{s}\left(\mathbb{R}^{d}\right)$ and $\mathscr{D} \cap F$ $H^{s}(\Omega)$ is dense in $F H^{s}(\Omega)$ for every $s \in \mathbb{R}$.

Definition 1. Given a real number $s$ and a $d \times d$ expansive matrix $M$, a nonzero closed linear subspace $X$ of $H^{s}\left(\mathbb{R}^{d}\right)$ is called a reducing subspace if $D X=X$ and $T_{k} X=X$ for each $k \in \mathbb{Z}^{d}$, and

$$
\left(1+\left|\left(M^{*}\right)^{-1} \cdot\right|^{2}\right)^{s / 2} \widehat{X}=\left(1+|\cdot|^{2}\right)^{s / 2} \widehat{X}
$$

where $\widehat{X}=\{\widehat{f}: f \in X\}$.

In particular, when $s=0,(12)$ is trivial and Definition 1 reduces to one in [4], which is characterized in Fourier domain as follows.
Proposition 2 ([4], (Theorem 1)). For a $d \times d$ expansive matrix $M, X$ is a reducing subspace of $L^{2}\left(\mathbb{R}^{d}\right)$ if and only if $X=F L^{2}(\Omega)$ for some $\Omega \subset \mathbb{R}^{d}$ with nonzero measure satisfying $\Omega=M^{*} \Omega$.

Proposition 3 ([4], (Theorem 2.1)). Let $s$ be a real number and $M a d \times d$ expansive matrix. Then, $X$ is a reducing subspace of $H^{s}\left(\mathbb{R}^{d}\right)$ if and only if $X=F H^{s}(\Omega)$ for some $\Omega \subset \mathbb{R}^{d}$ with nonzero measure satisfying $\Omega=M^{*} \Omega$.

So, to be specific, we denote a reducing subspace of $H^{s}($ $\mathbb{R}^{d}$ ) by $F H^{s}(\Omega)$ instead of $X$. Given a distribution $f$, we write

$$
\begin{gathered}
f_{j, k}(\cdot)=D^{j} T_{k} f(\cdot), \\
f_{j, k}^{s}(\cdot)=|\operatorname{det} M|^{-j s / d} f_{j, k}(\cdot),
\end{gathered}
$$

for $j \in \mathbb{Z}$ and $k \in \mathbb{Z}^{d}$. Let $F H^{s}(\Omega)$ and $F H^{-s}(\Omega)$ be reducing subspaces of $H^{s}\left(\mathbb{R}^{d}\right)$ and $H^{-s}\left(\mathbb{R}^{d}\right)$, respectively, and for $\psi_{0}$ $\in F H^{s}(\Omega), \tilde{\psi}_{0} \in F H^{-s}(\Omega), \Psi=\left\{\psi_{l}: 1 \leq l \leq L\right\}$, and $\tilde{\Psi}=\left\{\tilde{\psi}_{l}\right.$ $: 1 \leq l \leq L\}$ finite subsets of $F H^{s}(\Omega)$ and $F H^{-s}(\Omega)$, respectively, the wavelet systems $X^{s}\left(\psi_{0}, \Psi\right)$ and $X^{-s}\left(\tilde{\psi}_{0}, \tilde{\Psi}\right)$ are defined as

$$
\begin{array}{r}
X^{s}\left(\psi_{0}, \Psi\right):=\left\{\psi_{0,0, k}: k \in \mathbb{Z}^{d}\right\} \cup\left\{\psi_{l, j, k}^{s}: j \in N_{0}, k \in \mathbb{Z}^{d}, 1 \leq l \leq L\right\}, \\
X^{-s}\left(\tilde{\psi}_{0}, \tilde{\Psi}\right):=\left\{\tilde{\psi}_{0,0, k}: k \in \mathbb{Z}^{d}\right\} \cup\left\{\tilde{\psi}_{l, j, k}^{-s}: j \in N_{0}, k \in \mathbb{Z}^{d}, 1 \leq l \leq L\right\} .
\end{array}
$$

We say $\left(X^{s}\left(\psi_{0}, \Psi\right), X^{-s}\left(\tilde{\psi}_{0}, \tilde{\Psi}\right)\right)$ is a pair of dual wavelet frames for $\left(F H^{s}(\Omega), F H^{-s}(\Omega)\right)$ if

(1) $X^{s}\left(\psi_{0}, \Psi\right)$ is a wavelet frame for $F H^{s}(\Omega)$ and $X^{-s}($ $\left.\tilde{\psi}_{0}, \tilde{\Psi}\right)$ is a wavelet frame for $F H^{-s}(\Omega)$

(2) The identity

$$
\langle f, g\rangle=\sum_{k \in \mathbb{Z}^{d}}\left\langle f, \tilde{\psi}_{0,0, k}\right\rangle\left\langle\psi_{0,0, k}, g\right\rangle+\sum_{l=1}^{L} \sum_{j=0}^{\infty} \sum_{k \in \mathbb{Z}^{d}}\left\langle f, \tilde{\psi}_{l, j, k}^{-s}\right\rangle\left\langle\psi_{l, j, k}^{s}, g\right\rangle
$$

holds for $f \in F H^{s}(\Omega)$ and $g \in F H^{-s}(\Omega)$.

We say $\left(X^{s}\left(\psi_{0}, \Psi\right), X^{-s}\left(\tilde{\psi}_{0}, \tilde{\Psi}\right)\right)$ is a pair of weak dual wavelet frames for $\left(F H^{s}(\Omega), F H^{-s}(\Omega)\right)$ if

(1) $\left\{T_{k} \psi_{l}: k \in \mathbb{Z}^{d}, 0 \leq l \leq L\right\}$ and $\left\{T_{k} \tilde{\psi}_{l}: k \in \mathbb{Z}^{d}, 0 \leq l \leq\right.$ $L\}$ are Bessel sequences in $H^{s}\left(\mathbb{R}^{d}\right)$ and $H^{-s}\left(\mathbb{R}^{d}\right)$, respectively

(2) There exist dense subsets $V$ of $F H^{s}(\Omega)$ and $\tilde{V}$ of $F$ $H^{-s}(\Omega)$ such that 
$\langle f, g\rangle=\sum_{k \in \mathbb{Z}^{d}}\left\langle f, \tilde{\psi}_{0,0, k}\right\rangle\left\langle\psi_{0,0, k}, g\right\rangle+\sum_{l=1}^{L} \sum_{j=0}^{\infty} \sum_{k \in \mathbb{Z}^{d}}\left\langle f, \tilde{\psi}_{l, j, k}^{-s}\right\rangle\left\langle\psi_{l, j, k}^{s}, g\right\rangle$ forf $\in V, g \in \tilde{V}$,

where the series converges in the following sense: both $\sum_{k \in \mathbb{Z}^{d}}\left\langle f, \tilde{\psi}_{0,0, k}\right\rangle\left\langle\psi_{0,0, k}, g\right\rangle$ and $\sum_{k \in \mathbb{Z}^{d}}\left\langle f, \tilde{\psi}_{l, j, k}^{-s}\right\rangle\left\langle\psi_{l, j, k}^{s}, g\right\rangle$ converge unconditionally (equivalently, both $\sum_{k \in \mathbb{Z}^{d}} \mid\left\langle f, \tilde{\psi}_{0,0, k}\right\rangle\langle$ $\left.\psi_{0,0, k}, g\right\rangle \mid$ and $\sum_{k \in \mathbb{Z}^{d}}\left|\left\langle f, \tilde{\psi}_{l, j, k}^{-s}\right\rangle\left\langle\psi_{l, j, k}^{s}, g\right\rangle\right|$ converge), and

$$
\sum_{j=0}^{\infty} \sum_{k \in \mathbb{Z}^{d}}\left\langle f, \tilde{\psi}_{l, j, k}^{-s}\right\rangle\left\langle\psi_{l, j, k}^{s}, g\right\rangle=\lim _{J \longrightarrow \infty} \sum_{j=0}^{J} \sum_{k \in Z^{d}}\left\langle f, \tilde{\psi}_{l, j, k}^{-s}\right\rangle\left\langle\psi_{l, j, k}^{s}, g\right\rangle .
$$

It is obvious that the convergence of series in (16) is weaker than that in (15). And a pair of dual wavelet frames must be a pair of weak dual wavelet frames, whereas the converse is not true. Also, observe that in the above definition of weak dual wavelet frames, $\psi_{l}, 0 \leq l \leq L$ need not belong to $F H^{s}(\Omega)$, and $\tilde{\psi}_{l}, 0 \leq l \leq L$ need not belong to $F H^{-s}(\Omega)$.

Due to the great design freedom and the potential applications in signal denoising, image restoration, numerical analysis, etc., the study of wavelet frames for $L^{2}\left(\mathbb{R}^{d}\right)$ and Sobolev spaces has been attracting many researchers and seen great achievements (see [5-16] for details). In particular, Bownik in [6] obtained the following important characterization for homogeneous dual wavelet frames:

Proposition 4. Let $X(\Psi)$ and $X(\tilde{\Psi})$ be Bessel sequences in $L^{2}\left(\mathbb{R}^{d}\right)$. Then, $(X(\Psi), X(\tilde{\Psi}))$ is a pair of dual wavelet frames for $L^{2}\left(\mathbb{R}^{d}\right)$ if and only if

$$
\sum_{l=1}^{L} \sum_{j=-\infty}^{\kappa(k)} \widehat{\psi}_{l}\left(M^{*^{-j}} \cdot\right) \widehat{\psi}_{l}\left(M^{*^{-j}}(\cdot+k)\right)=\delta_{0, k} \text { a.e.on } \mathbb{R}^{d} \text { for } k \in \mathbb{Z}^{d},
$$

where $\kappa(k)$ is defined by Definition 6 in Section 2.

Li and Zhang in [17] generalized Proposition 4 to Sobolev space pairs $\left(H^{s}\left(\mathbb{R}^{d}\right), H^{-s}\left(R^{d}\right)\right)$ for nonhomogeneous dual wavelet frames:

Proposition 5. Given $s \in R$, let $X^{s}\left(\psi_{0}, \Psi\right)$ and $X^{-s}\left(\tilde{\psi}_{0}, \tilde{\Psi}\right)$ be Bessel sequences in $H^{s}\left(\mathbb{R}^{d}\right)$ and $H^{-s}\left(\mathbb{R}^{d}\right)$, respectively. Then, $\left(X^{s}\left(\psi_{0}, \Psi\right), X^{-s}\left(\tilde{\psi}_{0}, \tilde{\Psi}\right)\right)$ is a pair of dual frames in $\left(H^{s}\left(\mathbb{R}^{d}\right)\right.$ , $\left.H^{-s}\left(\mathbb{R}^{d}\right)\right)$ if and only if

$$
\widehat{\psi}_{0}(\cdot) \widehat{\hat{\psi}}_{0}(\cdot+k)+\sum_{l=1}^{L} \sum_{j=0}^{k(k)} \widehat{\psi}_{l}\left(M^{*-j} \cdot\right) \widehat{\psi}_{l}\left(M^{*-j}(+k)\right)=\delta_{0, k} \text { a.e.on } \mathbb{R}^{d} \text { for } k \in \mathbb{Z}^{d} .
$$

An important method to construct (dual) wavelet frames from refinable functions is extension principles. Ron and Shen in $[15,16]$ prosed the unitary extension principle (UEP) and the mixed extension principle (MEP). Subse- quently, Daubechies et al. in [10] developed them in the form of the oblique extension principle (OEP) and the mixed oblique extension principle (MOEP). From then on, the study of the extension principles has interested many researchers $[4,5,7,8,11,18-20]$.

Observe that all above works, the wavelet systems (or the refinable functions) are required to be Bessel sequences. In order to achieve the Bessel property, some conditions have to be imposed on the wavelet systems (or the refinable functions) that are too technical and artificial. It is natural to ask what are expected from general refinable functions without too many restrictions. For this purpose, Jia and $\mathrm{Li}$ in [21] introduced the nation of weak wavelet biframes (weak dual wavelet frames). Starting from a pair of general refinable functions without smoothness restrictions, they obtained a construction of weak dual wavelet frames for reducing subspace $F L^{2}(\Omega)$ of $L^{2}\left(\mathbb{R}^{d}\right)$.

Inspired by all these works, in present paper, we investigate a class of weak dual wavelet frames for reducing subspaces of Sobolev spaces. In Section 2, we first give some necessary lemmas, and then, we give a Fourier-domain characterization of weak dual wavelet frames in $\left(F H^{s}(\Omega), F H^{-s}(\Omega)\right)$ associated with $\left(D \cap F H^{s}(\Omega), D \cap F H^{-s}(\Omega)\right)$. In Section 3, by using the above characterization, we derive a mixed oblique extension principle for such weak dual wavelet frames.

Before proceeding, we introduce some necessary notations. We denote by $T^{d}=[0,1)^{d}$ the $d$-dimensional torus and, for a Lebesgue measurable set $E$ in $\mathbb{R}^{d}$, by $|E|$ its Lebesgue measure and $\chi_{E}$ its characteristic function, respectively, by $\delta$ the Dirac sequence such that $\delta_{0,0}=1$ and $\delta_{0, k}=0$ for $0 \neq k \epsilon$ $\mathbb{Z}^{d}$, and denote by $\tau$ the mapping from $\mathbb{R}^{d}$ to $\mathbb{T}^{d}$ defined by

$$
\tau(x)=x-k \text { for } x \in \mathbb{T}^{d}+k \text { with } k \in \mathbb{Z}^{d} .
$$

For functions $f$ and $g$ on $\mathbb{Z}^{d}$, we define

$$
[f, g]_{t}(\cdot)=\sum_{k \in \mathbb{Z}^{d}} f(\cdot+k) g(\cdot+k)\left(1+|\cdot+k|^{2}\right)^{t}, t \in \mathbb{R},
$$

if it is well defined, and the spectrum $\sigma_{t}(f)$ by

$$
\sigma_{t}(f)=\left\{\xi \in T^{d}:[\widehat{f}, \widehat{f}]_{t}(\xi)>0\right\} .
$$

It is obvious that $\xi \in \sigma_{t}(f)$ if and only if $\xi \in T^{d}$ and $\widehat{f}(\xi$ $+k) \neq 0$ for some $k \in Z^{d}$. So $\sigma_{t}(f)$ is independent of $t$. For simplicity, we use $\sigma(f)$ to replace $\sigma_{t}(f)$.

\section{The Characterization of Weak Dual Wavelet Frames}

This section is devoted to characterizing weak dual wavelet frames in $\left(F H^{s}(\Omega), F H^{-s}(\Omega)\right)$. Fist, we give some necessary lemmas for later use. 
Definition 6. Let $M$ be a $d \times d$ expansive matrix. Define a function $\kappa: \mathbb{Z}^{d} \longrightarrow N_{0}$ by

$$
\kappa(n)=\sup \left\{j \in N_{0}: M^{*^{-j}} n \in \mathbb{Z}^{d}\right\}
$$

and set $\kappa(0)=+\infty$.

By a standard argument, we have the following three lemmas:

Lemma 7. $\left\{M^{*^{-\kappa(n)-1}} n: 0 \neq n \in \mathbb{Z}^{d}\right\}=\bigcup_{\gamma \in \Gamma_{M^{*}} \backslash\{0\}}\left(\mathbb{Z}^{d}+\gamma\right)$.

Lemma 8. For $\phi \in H^{s}\left(\mathbb{R}^{d}\right)$ and $\widetilde{\phi} \in H^{-s}\left(\mathbb{R}^{d}\right)$, we have

$\sigma(\phi) \cap \tau(\sigma(\widetilde{\phi})-\gamma)=\left\{\xi \in T^{d}: \widehat{\phi}(\xi+k) \widehat{\widetilde{\phi}}(\xi+\gamma+n) \neq 0\right.$ for some $\left.k, n \in \mathbb{Z}^{d}\right\}$ for $\gamma \in \Gamma_{M^{*}}$

Lemma 9. Let $\left\{a_{k}\right\}_{k \in \mathbb{Z}^{d}}$ and $\left\{b_{k}\right\}_{k \in \mathbb{Z}^{d}}$ be two complex sequences, and $\sum_{j=0}^{\infty} \sum_{0 \neq k \in \mathbb{Z}^{d}}\left|a_{M^{* j}{ }^{j}} b_{k}\right|<\infty$. Then,

$$
\sum_{j=0}^{\infty} \sum_{0 \neq k \in \mathbb{Z}^{d}} a_{M^{* j} k} b_{k}=\sum_{0 \neq k \in \mathbb{Z}^{d}} \sum_{j=0}^{\kappa(k)} a_{k} b_{M^{*-j} k}
$$

Lemma 10 ([4], (Lemma 3.1)). Given $s \in R$, a $d \times d$ expansive matrix $M$, and $\phi \in H^{s}\left(R^{d}\right)$, we have the following:

(i) $\left\{T_{k} \phi: k \in \mathbb{Z}^{d}\right\}$ is a Bessel sequence in $H^{s}\left(\mathbb{R}^{d}\right)$ if and only if $[\widehat{\phi}, \widehat{\phi}]_{s} \in L^{\infty}\left(\mathbb{R}^{d}\right)$. In this case, $\|\widehat{\phi}, \widehat{\phi}\|_{L^{\infty}\left(\mathbb{R}^{d}\right)}$ is a Bessel bound

(ii) If $\left\{T_{k} \phi: k \in Z^{d}\right\}$ is a Bessel sequence in $H^{s}\left(\mathbb{R}^{d}\right)$, then $\left\{\phi_{n, k}: k \in \mathbb{Z}^{d}\right\}$ is a Bessel sequence in $H^{s}\left(\mathbb{R}^{d}\right)$ with the Bessel bound $C_{n}\left\|[\widehat{\phi}, \widehat{\phi}]_{s}\right\|_{L^{\infty}\left(\mathbb{T}^{d}\right)}$ for $n \in Z$, and

$$
\sum_{k \in \mathbb{Z}^{d}}\left|\left\langle f, \phi_{n, k}\right\rangle\right|^{2} \leq C_{n}\left\|[\widehat{\phi}, \widehat{\phi}]_{s}\right\|_{L^{\infty}\left(\mathbb{T}^{d}\right)}\|f\|_{H^{-s}\left(\mathbb{R}^{d}\right)}^{2}
$$

for $f \in H^{-s}\left(\mathbb{R}^{d}\right)$, where $C_{n}=\sup _{\xi \in R^{d}}\left(1+|\xi|^{2} / 1+\left|M^{*^{-n}} \xi\right|^{2}\right)^{s}$.

Lemma 11. Let $s \in R, f \in H^{s}\left(\mathbb{R}^{d}\right), \psi \in H^{-s}\left(\mathbb{R}^{d}\right)$, and $j \in Z$. Then, for $k \in Z^{d}$, the $k$-th Fourier coefficient of $\left[|\operatorname{det} M|^{j / 2} \widehat{f}\left(M^{*^{j}} \cdot\right), \widehat{\psi}(\cdot)\right]_{0}(\xi)$ is $\left\langle f, \psi_{j, k}\right\rangle$. In particular,

$$
\left[|\operatorname{det} M|^{j / 2} \widehat{f}\left(M^{*^{j}} \cdot\right), \widehat{\psi}(\cdot)\right]_{0}(\xi)=\sum_{k \in \mathbb{Z}^{d}}\left\langle f, \psi_{j, k}\right\rangle e^{2 \pi i\langle k, \xi\rangle},
$$

if $\left\{T_{k} \psi: k \in \mathbb{Z}^{d}\right\}$ is a Bessel sequence in $H^{-s}\left(\mathbb{R}^{d}\right)$.
Proof. Since $f \in H^{s}\left(\mathbb{R}^{d}\right)$ and $\psi \in H^{-s}\left(\mathbb{R}^{d}\right)$, we have $\widehat{f}\left(M^{*^{j}}\right.$. )$\widehat{\psi}(\cdot) \in L^{1}\left(\mathbb{R}^{d}\right)$, and thus,

$$
\begin{aligned}
\int_{\mathbb{R}^{d}} & {\left[|\operatorname{det} M|^{j / 2} \widehat{f}\left(M^{*^{j}} \cdot\right), \widehat{\psi}(\cdot)\right]_{0}(\xi) e^{-2 \pi i\langle k, \xi\rangle} d \xi } \\
= & \left.\operatorname{det} M\right|^{j / 2} \int_{\mathbb{R}^{d}} \widehat{f}\left(M^{*^{j}} \xi\right) \widehat{\psi}(\xi) e^{-2 \pi i\langle k, \xi\rangle} d \xi \\
= & \\
= & \left.\left.\operatorname{det} M\right|^{-j / 2} \int_{\mathbb{R}^{d}} \widehat{f}(\xi) \widehat{\psi}\left(M^{*^{-j}} \xi\right) e^{-2 \pi i\left\langle k, M^{*} \xi\right.} \xi\right\rangle \xi \\
= & \int_{\mathbb{R}^{d}} \widehat{f}(\xi)\left[\widehat{\left.\psi_{j, k}(\cdot)\right]}(\xi) d \xi=\left\langle f, \psi_{j, k}\right\rangle,\right.
\end{aligned}
$$

by the Plancherel theorem. So the $k$-th Fourier coefficient of $\left[|\operatorname{det} M|^{j / 2} \widehat{f}\left(M^{*^{j}} \cdot\right), \widehat{\psi}(\cdot)\right]_{0}(\xi)$ is $\left\langle f, \psi_{j, k}\right\rangle$.

If $\left\{T_{k} \psi: k \in \mathbb{Z}^{d}\right\}$ is a Bessel sequence in $H^{-s}\left(\mathbb{R}^{d}\right)$, then $\left\{\psi_{j, k}: k \in \mathbb{Z}^{d}\right\}$ is a Bessel sequence in $H^{-s}\left(\mathbb{R}^{d}\right)$ by Lemma 10 (ii). It follows that $\left\{\left\langle f, \psi_{j, k}\right\rangle\right\}_{k \in \mathbb{Z}^{d}} \in \ell^{2}\left(\mathbb{Z}^{d}\right)$, and thus, (27) holds.

Lemma 12 ([4], (Lemma 3.5)). Let $S$ be a bounded set in $\mathbb{R}^{d}$. Then, there exist finite sets $F_{1} \subset N_{0}$ and $F_{2} \subset Z^{d} \backslash\{0\}$ such that

$S \cap\left(S+M^{* j} k\right)=\varnothing$ for $(j, k) \in\left(N_{0} \times\left(Z^{d} \backslash\{0\}\right)\right) \backslash F_{1} \times F_{2}$.

Lemma 13. Given $s \in \mathbb{R}$, let $\left\{T_{k} \psi_{l}: k \in \mathbb{Z}^{d}, 0 \leq l \leq L\right\}$ and \{ $\left.T_{k} \tilde{\psi}_{l}: k \in \mathbb{Z}^{d}, 0 \leq l \leq L\right\}$ be Bessel sequences in $H^{s}\left(\mathbb{R}^{d}\right)$ and $H^{-s}\left(\mathbb{R}^{d}\right)$, respectively. Then,

$$
\begin{aligned}
& \sum_{k \in \mathbb{Z}^{d}}\left\langle f, \tilde{\psi}_{0}(\cdot-k)\right\rangle\left\langle\psi_{0}(\cdot-k), g\right\rangle+\sum_{l=1}^{L} \sum_{j=0}^{\infty} \sum_{k \in \mathbb{Z}^{d}}\left\langle f, \tilde{\psi}_{l, j, k}^{-s}\right\rangle\left\langle\psi_{l, j, k}^{s}, g\right\rangle
\end{aligned}
$$

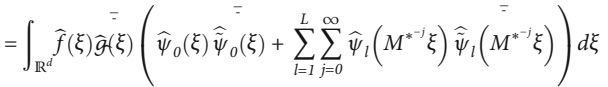

$$
\begin{aligned}
& +\int_{\mathbb{R}^{d}} \bar{g}(\xi) \sum_{0 \neq k \in \mathbb{Z}^{d}} \hat{f}(\xi+k)\left(\widehat{\psi}_{0}(\xi) \widehat{\tilde{\psi}}_{0}(\xi+k)+\sum_{l=1}^{L} \sum_{j=0}^{k(k)} \widehat{\psi}_{l}\left(M^{*-j} \xi\right) \widehat{\tilde{\psi}}_{l}\left(M^{*^{-j}}(\xi+k)\right)\right) d \xi,
\end{aligned}
$$

for $f, g \in \mathscr{D}$, where $\mathscr{D}$ is defined by (11).

Proof. By Lemma 11, we have

$$
\begin{aligned}
\sum_{k \in \mathbb{Z}^{d}}\left\langle f, \tilde{\psi}_{0}(\cdot-k)\right\rangle\left\langle\psi_{0}(\cdot-k), g\right\rangle+\sum_{l=1}^{L} \sum_{j=0}^{\infty} \sum_{k \in \mathbb{Z}^{d}}\left\langle f, \tilde{\psi}_{l, j, k}^{-s}\right\rangle\left\langle\psi_{l, j, k}^{s}, g\right\rangle \\
=\int_{\mathbb{T}^{d}}\left(\sum_{k \in \mathbb{Z}^{d}} \widehat{f}(\xi+k) \widehat{\tilde{\psi}}_{0}(\xi+k)\right)\left(\sum_{k \in \mathbb{Z}^{d}} \widehat{\psi}_{0}(\xi+k) \widehat{\xi}(\xi+k)\right) d \xi \\
\quad+\sum_{l=1}^{L} \sum_{j=0}^{\infty}|\operatorname{det} M|^{j} \int_{\mathbb{J}^{d}}\left(\sum_{k \in \mathbb{Z}^{d}} \hat{f}\left(M^{*^{j}}(\xi+k)\right) \widehat{\tilde{\psi}}_{l}(\xi+k)\right) \\
\quad \times\left(\sum_{k \in \mathbb{Z}^{d}} \widehat{\psi}_{l}(\xi+k) \hat{\mathcal{g}}\left(M^{*^{j}}(\xi+k)\right)\right) d \xi .
\end{aligned}
$$


Write

$$
\begin{gathered}
G(\cdot)=\sum_{k \in \mathbb{Z}^{d}} \widehat{\psi}_{0}(\cdot+k) \widehat{\mathscr{G}}(\cdot+k), \\
G_{l, j}(\cdot)=\sum_{k \in \mathbb{Z}^{d}} \widehat{\psi}_{l}(\cdot+k) \hat{\mathcal{G}}\left(M^{*^{j}}(\cdot+k)\right) .
\end{gathered}
$$

Then, by the Cauchy-Schwarz inequality, we have

$$
\begin{aligned}
|G(\cdot)| \leq \sum_{k \in \mathbb{Z}^{d}}\left|\widehat{\psi}_{0}(\cdot+k) \hat{g}(\cdot+k)\right| \leq\left(\sum_{k \in \mathbb{Z}^{d}}\left|\psi \wedge_{0}(\cdot+k)\right|^{2}\left(1+|\cdot+k|^{2}\right)^{s}\right)^{1 / 2} \\
\cdot\left(\sum_{k \in \mathbb{Z}^{d}}|g \wedge(\cdot+k)|^{2}\left(1+|\cdot+k|^{2}\right)^{-s}\right)^{1 / 2}=\left[\widehat{\psi}_{0}, \widehat{\psi}_{0}\right]_{s}^{1 / 2}(\cdot)[\hat{g}, \widehat{g}]_{-s}^{1 / 2}(\cdot) .
\end{aligned}
$$

By a similar procedure, we also have

$$
\left|G_{l, j}(\cdot)\right| \leq\left[\widehat{\psi}_{l}, \widehat{\psi}_{l}\right]_{s}^{1 / 2}(\cdot)\left[\hat{g}\left(M^{*^{j}} \cdot\right), \hat{g}\left(M^{*^{j}} \cdot\right)\right]_{-s}^{1 / 2}(\cdot) .
$$

If $g \in D$, then $\left\{T_{k} g: k \in \mathbb{Z}^{d}\right\}$ and $\left\{T_{k} \tilde{g}: k \in \mathbb{Z}^{d}\right\}$ with $\widehat{\tilde{g}}(\cdot)=\widehat{g}\left(M^{* j} \cdot\right)$ are Bessel sequences in $H^{-s}\left(\mathbb{R}^{d}\right)$. Also, observe that $\left\{T_{k} \psi_{0}: k \in \mathbb{Z}^{d}\right\}$ and $\left\{T_{k} \psi_{l}: k \in \mathbb{Z}^{d}, 1 \leq l \leq L\right\}$ are Bessel sequences in $H^{s}\left(\mathbb{R}^{d}\right)$, and thus, $G, G_{l, j} \in L^{\infty}\left(\mathbb{T}^{d}\right)$ by Lemma 10 (i). It follows that

$$
\begin{aligned}
& \int_{T^{d}}\left(\sum_{k \in \mathbb{Z}^{d}} \hat{f}(\xi+k) \widehat{\tilde{\psi}}_{0}(\xi+k)\right)\left(\sum_{k \in \mathbb{Z}^{d}} \widehat{\psi}_{0}(\xi+k) \hat{\mathcal{g}}(\xi+k)\right) d \xi \\
& =\int_{\mathbb{R}^{d}} \widehat{\psi}_{0}(\xi) \hat{\mathcal{g}}(\xi)\left(\sum_{k \in \mathbb{Z}^{d}} \hat{f}(\xi+k) \widehat{\tilde{\psi}}_{0}(\xi+k)\right) d \xi, \\
& \int_{T^{d}}\left(\sum_{k \in \mathbb{Z}^{d}} \widehat{f}\left(M^{*^{j}}(\xi+k)\right) \widehat{\tilde{\psi}}_{l}(\xi+k)\right)\left(\sum_{k \in \mathbb{Z}^{d}} \widehat{\psi}_{l}(\xi+k) \hat{g}\left(M^{*^{j}}(\xi+k)\right)\right) d \xi \\
& =\int_{\mathbb{R}^{d}} \widehat{\psi}_{l}(\xi) \hat{\mathcal{g}}\left(M^{* j} \xi\right)\left(\sum_{k \in \mathbb{Z}^{d}} \hat{f}\left(M^{*^{j}}(\xi+k)\right) \widehat{\tilde{\psi}}_{l}(\xi+k)\right) d \xi,
\end{aligned}
$$

by the Fubini-Tonelli theorem. Therefore, we get

$$
\begin{aligned}
& \sum_{k \in \mathbb{Z}^{d}}\left\langle f, \tilde{\psi}_{0}(\cdot-k)\right\rangle\left\langle\psi_{0}(-k), g\right\rangle+\sum_{l=1}^{L} \sum_{j=0}^{\infty} \sum_{k \in \mathbb{Z}^{d}}\left\langle f, \tilde{\psi}_{l, j, k}^{-s}\right\rangle\left\langle\psi_{l, j, k}^{s}, g\right\rangle \\
& =\int_{\mathbb{R}^{d}} \widehat{\psi}_{0}(\xi) \overline{\mathcal{g}}(\xi)\left(\sum_{k \in \mathbb{Z}^{d}} \hat{f}(\xi+k) \widehat{\hat{\psi}}_{0}(\xi+k)\right) d \xi \\
& \quad+\sum_{l=1}^{L} \sum_{j=0}^{\infty}|\operatorname{det} M|^{j} \int_{\mathbb{R}^{d}} \widehat{\psi}_{l}(\xi) \hat{g}\left(M^{* j} \xi\right)\left(\sum_{k \in \mathbb{Z}^{d}} \widehat{\hat{f}}\left(M^{* j}(\xi+k)\right) \widehat{\tilde{\psi}}_{l}(\xi+k)\right) d \xi .
\end{aligned}
$$

By using the Cauchy-Schwarz inequality, we have

$$
\begin{aligned}
& \left|\widehat{\psi}_{0}(\cdot) \overline{\dot{g}(\cdot)}\right|\left(\sum_{k \in \mathbb{Z}^{d}}\left|\widehat{f}(\cdot+k) \underset{\hat{\Psi}_{0}(\cdot+k) \mid}{\overline{(}}\right|\right) \\
& \leq\left(\sum_{k \in \mathbb{Z}^{d}} \mid \widehat{\psi}_{0}(\cdot+k) \hat{\mathscr{g}(\cdot+k)}=\right)\left(\sum_{k \in \mathbb{Z}^{d}}\left|\widehat{f}(\cdot+k) \widehat{\tilde{\psi}}_{0}(\cdot+k)\right|\right) \\
& \leq\left(\sum_{k \in \mathbb{Z}^{d}}\left|\psi \wedge_{0}(\cdot+k)\right|^{2}\left(1+|\cdot+k|^{2}\right)^{s}\right)^{1 / 2}\left(\sum_{k \in \mathbb{Z}^{d}}|g \wedge(++k)|^{2}\left(1+|\cdot+k|^{2}\right)^{-s}\right)^{1 / 2} \\
& \times\left(\sum_{k \in \mathbb{Z}^{d}}|f \wedge(++k)|^{2}\left(1+|+k|^{2}\right)^{s}\right)^{1 / 2}\left(\sum_{k \in \mathbb{Z}^{d}}\left|\tilde{\psi} \wedge_{0}(++k)\right|^{2}\left(1+|++k|^{2}\right)^{-s}\right)^{1 / 2} \\
& =\left[\widehat{\psi}_{0}, \widehat{\psi}_{0}\right]_{s}^{1 / 2}(\cdot)\left[\hat{g}, \widehat{g}_{-s}^{1 / 2}(\cdot)[\widehat{f}, \hat{f}]_{s}^{1 / 2}(\cdot)\left[\widehat{\hat{\psi}}_{0}, \widehat{\tilde{\psi}}_{0}\right]_{-s}^{1 / 2}(\cdot),\right.
\end{aligned}
$$

which belongs to $L^{\infty}\left(R^{d}\right)$ by Lemma 10 (i) since $\left\{T_{k} f: k\right.$ $\left.\in Z^{d}\right\}$ is also a Bessel sequence in $H^{s}\left(R^{d}\right)$ if $f \in D$, and thus,

$$
\begin{aligned}
& \int_{R^{d}} \widehat{\psi}_{0}(\xi) \hat{g}(\xi)\left(\sum_{k \in Z^{d}} \hat{f}(\xi+k) \widehat{\tilde{\psi}}_{0}(\xi+k)\right) d \xi \\
& =\int_{R^{d}} \widehat{\psi}_{0}(\xi) \hat{g}(\xi) \widehat{f}(\xi) \widehat{\tilde{\psi}}_{0}(\xi) d \xi+\int_{R^{d}} \widehat{\psi}_{0}(\xi) \hat{g}(\xi) \sum_{0 \neq k \in Z^{d}} \hat{f}(\xi+k) \widehat{\tilde{\psi}}_{0}(\xi+k) d \xi .
\end{aligned}
$$

Since $f, g \in D$, then there exists a bounded set $S$ in $R^{d}$ such that $\operatorname{supp}(\widehat{f}) \cup \operatorname{supp}(\widehat{g}) \subset S$. By Lemma 12, there exist finite sets $F_{1} \subset N_{0}$ and $F_{2} \subset Z^{d} \backslash\{0\}$ such that

$$
S \cap\left(S+M^{*^{j}} k\right)=\varnothing \text { for }(j, k) \in\left(N_{0} \times\left(Z^{d} \backslash\{0\}\right)\right) \backslash F_{1} \times F_{2}
$$

Therefore, we have

$$
\begin{gathered}
\sum_{l=1}^{L} \sum_{j=0}^{\infty}|\operatorname{det} M|^{j} \int_{R^{d}} \widehat{\psi}_{l}(\xi) \hat{g}\left(M^{* j} \xi\right)\left(\sum_{0 \neq k \in Z^{d}} \widehat{f}\left(M^{*^{j}}(\xi+k)\right) \widehat{\tilde{\psi}}_{l}(\xi+k)\right) d \xi \\
=\sum_{l=1}^{L} \sum_{j \in F_{1}}|\operatorname{det} M|^{j} \int_{R^{d}} \widehat{\psi}_{l}(\xi) \hat{\mathcal{g}}\left(M^{* j} \xi\right)\left(\sum_{k \in F_{2}} \widehat{f}\left(M^{* j}(\xi+k)\right) \widehat{\hat{\psi}}_{l}(\xi+k)\right) d \xi .
\end{gathered}
$$

Write

$$
K=\bigcup_{k \in F_{2} \cup\{0\}}\left(\cup_{j \in F_{1}} M^{*^{-j}} S-k\right) .
$$


Then, for $j \in F_{1}$ and $k \in F_{2}$, we have

$$
\begin{aligned}
& |\operatorname{det} M|^{j} \int_{R^{d}}\left|\widehat{\psi}_{l}(\xi) \hat{\mathcal{g}}\left(M^{* j} \xi\right) \hat{f}\left(M^{*^{j}}(\xi+k)\right) \widehat{\tilde{\psi}}_{l}(\xi+k)\right| d \xi \\
& \quad=\int_{R^{d}}\left|\widehat{\psi}_{l}\left(M^{*^{-j}} \xi\right) \hat{\mathfrak{g}}(\xi) \hat{f}\left(\xi+M^{*^{j}} k\right) \widehat{\tilde{\psi}}_{l}\left(M^{*^{-j}} \xi+k\right)\right| d \xi \\
& \quad \leq\|\hat{\mathfrak{g}}\|_{L^{\infty}\left(R^{d}\right)}\|\widehat{f}\|_{L^{\infty}\left(R^{d}\right)}\left(\int_{M^{*-j} S}\left|\psi \wedge_{l}(\xi)\right|^{2} d \xi\right)^{1 / 2}\left(\int_{M^{*^{-j}} S}\left|\tilde{\psi} \wedge_{l}(\xi+k)\right|^{2} d \xi\right)^{1 / 2} \\
& \quad \leq\|\hat{\mathfrak{g}}\|_{L^{\infty}\left(R^{d}\right)}\|\widehat{f}\|_{L^{\infty}\left(R^{d}\right)}\left(\int_{K}\left|\psi \wedge_{l}(\xi)\right|^{2} d \xi\right)^{1 / 2}\left(\int_{K}\left|\tilde{\psi} \wedge_{l}(\xi)\right|^{2} d \xi\right)^{1 / 2} .
\end{aligned}
$$

Also, observe that $\left.\max _{\xi \in K}\left(1+|\xi|^{2}\right)\right)^{-s / 2}\left(1+|\xi|^{2}\right)^{s / 2} \geq 1$ and $\left(\max _{\xi \in K}\left(1+|\xi|^{2}\right)\right)^{s / 2}\left(1+|\xi|^{2}\right)^{-s / 2} \geq 1$. It follows that

$$
\begin{aligned}
& |\operatorname{det} M|^{j} \int_{R^{d}}\left|\widehat{\psi}_{l}(\xi) \hat{\mathfrak{g}}\left(M^{*^{j}} \xi\right) \hat{f}\left(M^{*^{j}}(\xi+k)\right) \widehat{\tilde{\psi}}_{l}(\xi+k)\right| d \xi \\
& \leq\|\widehat{\hat{g}}\|_{L^{\infty}\left(R^{d}\right)}\|\widehat{f}\|_{L^{\infty}\left(R^{d}\right)}\left(\max _{\xi \in K}\left(1+|\xi|^{2}\right)\right)^{-s / 2}\left(\max _{\xi \in K}\left(1+|\xi|^{2}\right)\right)^{s / 2} \\
& \quad \times\left(\int_{K}\left|\psi \wedge_{l}(\xi)\right|^{2}\left(1+|\xi|^{2}\right)^{s} d \xi\right)^{1 / 2} \times\left(\int_{K}\left|\tilde{\psi} \wedge_{l}(\xi)\right|^{2}\left(1+|\xi|^{2}\right)^{-s} d \xi\right)^{1 / 2} \\
& \leq\left\|\widehat{g}_{L^{\infty}}\left(R^{d}\right)\right\| \widehat{f} \|_{L^{\infty}\left(R^{d}\right)}\left(\max _{\xi \in K}\left(1+|\xi|^{2}\right)\right)^{-s / 2} \\
& \quad \times\left(\max _{\xi \in K}\left(1+|\xi|^{2}\right)\right)^{s / 2}\left\|\widehat{\psi}_{l}\right\|_{H^{s}\left(R^{d}\right)}\left\|\widehat{\tilde{\psi}}_{l}\right\|_{H^{-s}\left(R^{d}\right)}<\infty .
\end{aligned}
$$

Therefore, we can change the order of summation and integration in (40).

$$
\begin{aligned}
& \sum_{l=1}^{L} \sum_{j=0}^{\infty}|\operatorname{det} M|^{j} \int_{R^{d}} \widehat{\psi}_{l}(\xi) \hat{\mathcal{g}}\left(M^{*^{j}} \xi\right)\left(\sum_{0 \neq k \in Z^{d}} \hat{f}\left(M^{*^{j}}(\xi+k)\right) \widehat{\tilde{\psi}}_{l}(\xi+k)\right) d \xi \\
& =\sum_{l=1}^{L} \sum_{j=0}^{\infty} \int_{R^{d}} \widehat{\psi}_{l}\left(M^{*^{-j}} \xi\right) \hat{\mathcal{G}}(\xi)\left(\sum_{0 \neq k \in Z^{d}} \hat{f}\left(\xi+M^{*^{j}} k\right) \widehat{\tilde{\psi}}_{l}\left(M^{*^{-j}} \xi+k\right)\right) d \xi \\
& =\int_{R^{d}} \sum_{l=1}^{L} \sum_{j=0}^{\infty} \widehat{\psi}_{l}\left(M^{*^{-j}} \xi\right) \hat{\bar{g}}(\xi)\left(\sum_{0 \neq k \in Z^{d}} \widehat{f}\left(\xi+M^{* j} k\right) \widehat{\tilde{\psi}}_{l}\left(M^{*^{-j}} \xi+k\right)\right) d \xi \\
& =\int_{R^{d}} \widehat{\bar{g}}(\xi) \sum_{0 \neq k \in Z^{d}} \widehat{f}(\xi+k) \sum_{l=1}^{L} \sum_{j=0}^{\kappa(k)} \widehat{\psi}_{l}\left(M^{*^{-j}} \xi\right) \widehat{\tilde{\psi}}_{l}\left(M^{*^{-j}}(\xi+k)\right) d \xi,
\end{aligned}
$$

where Lemma 9 is used in the last equality. And thus, the conclusion follows by collecting (36), (38), and (44). $\square \square \square$

The following theorem characterizes a pair of weak dual wavelet frames for $\left(F H^{s}(\Omega), F H^{-s}(\Omega)\right)$.

Theorem 14. Let $F H^{s}(\Omega)$ and $F H^{-s}(\Omega)$ be reducing subspaces of $H^{s}\left(R^{d}\right)$ and $H^{-s}\left(R^{d}\right)$, respectively, and $\psi_{0} \in F H^{s}($ $\Omega), \quad \tilde{\psi}_{0} \in F H^{-s}(\Omega)$ and $\Psi=\left\{\psi_{l}: 1 \leq l \leq L\right\}, \quad \tilde{\Psi}=\left\{\tilde{\psi}_{l}: 1 \leq l\right.$ $\leq L\}$ finite subsets of $F H^{s}(\Omega)$ and $F H^{-s}(\Omega)$. Suppose $\left\{T_{k}\right.$ $\left.\psi_{l}: k \in Z^{d}, 0 \leq l \leq L\right\}$ and $\left\{T_{k} \tilde{\psi}_{l}: k \in Z^{d}, 0 \leq l \leq L\right\}$ are both Bessel sequences in $H^{s}\left(R^{d}\right)$ and $H^{-s}\left(R^{d}\right)$, respectively. Then,
$\left(X^{s}\left(\psi_{0}, \Psi\right), X^{-s}\left(\tilde{\psi}_{0}, \tilde{\Psi}\right)\right)$ is a pair of weak dual wavelet frames for $\left(F H^{s}(\Omega), F H^{-s}(\Omega)\right)$ if and only if

$$
\begin{aligned}
& \lim _{J \longrightarrow \infty} \int_{R^{d}}\left(\widehat{\psi}_{0}(\xi) \widehat{\tilde{\psi}}_{0}(\xi)+\sum_{l=1}^{L} \sum_{j=0}^{J} \widehat{\psi}_{l}\left(M^{*^{-j}} \xi\right) \widehat{\tilde{\psi}}_{l}\left(M^{*^{-j}} \xi\right)\right) h(\xi) d \xi \\
& =\int_{R^{d}} \chi_{\Omega}(\xi) h(\xi) d \xi \text { for } h \in L^{\infty}(K), \forall \text { compact } K \subset R^{d} \backslash\{0\} \\
& = \\
& \widehat{\psi}_{0}(\cdot) \widehat{\tilde{\psi}}_{0}(+k)+\sum_{l=1}^{L} \sum_{j=0}^{k(k)} \widehat{\psi}_{l}\left(M^{*^{-j}} \cdot\right) \widehat{\tilde{\psi}}_{l}\left(M^{*^{-j}}(\cdot+k)\right) \\
& =0 \text { for } 0 \neq k \in Z^{d} \text { and a.e.on } \Omega .
\end{aligned}
$$

Proof. Since $D \cap F H^{s}(\Omega)$ is dense in $F H^{s}(\Omega)$ for every $s \in R$, then $\left(X^{s}\left(\psi_{0}, \Psi\right), X^{-s}\left(\tilde{\psi}_{0}, \tilde{\Psi}\right)\right)$ is a pair of weak dual wavelet frames for $\left(F H^{s}(\Omega), F H^{-s}(\Omega)\right)$ if and only if

$$
\sum_{k \in Z^{d}}\left\langle f, \tilde{\psi}_{0,0, k}\right\rangle\left\langle\psi_{0,0, k}, g\right\rangle+\sum_{l=1}^{L} \sum_{j=0}^{\infty} \sum_{k \in Z^{d}}\left\langle f, \tilde{\psi}_{l, j, k}^{-s}\right\rangle\left\langle\psi_{l, j, k}^{s}, g\right\rangle=\langle f, g\rangle,
$$

for $f \in D \cap F H^{s}(\Omega), g \in D \cap F H^{-s}(\Omega)$, or equivalently

$$
\begin{aligned}
& \sum_{k \in Z^{d}}\left\langle\left(f \wedge \chi_{\Omega}\right)^{\vee}, \tilde{\psi}_{0,0, k}\right\rangle\left\langle\psi_{0,0, k},\left(g \wedge \chi_{\Omega}\right)^{\vee}\right\rangle+\sum_{l=1}^{L} \sum_{j=0}^{\infty} \sum_{k \in Z^{d}}\left\langle\left(f \wedge \chi_{\Omega}\right)^{\vee}, \tilde{\psi}_{l, j, k}^{-s}\right\rangle\left\langle\psi_{l, j, k}^{s},\left(g \wedge \chi_{\Omega}\right)^{\vee}\right\rangle \\
& =\left\langle\left(f \wedge \chi_{\Omega}\right)^{\vee},\left(g \wedge \chi_{\Omega}\right)^{\vee}\right\rangle=\left\langle\hat{f} \chi_{\Omega}, \widehat{g} \chi_{\Omega}\right\rangle,
\end{aligned}
$$

for $f, g \in D$ due to $D \cap F H^{s}(\Omega)=\left\{\left(h \wedge \chi_{\Omega}\right)^{\vee}: h \in D\right\}$. By the Bessel assumptions, we know that the series $\sum_{k \in Z^{d}}\langle$ $\left.\left(f \wedge \chi_{\Omega}\right)^{\vee}, \tilde{\psi}_{0,0, k}\right\rangle\left\langle\psi_{0,0, k},\left(g \wedge \chi_{\Omega}\right)^{\vee}\right\rangle$ and $\sum_{k \in Z^{d}}\left\langle\left(f \wedge \chi_{\Omega}\right)^{\vee}, \tilde{\psi}_{l, j, k}^{-s}\right\rangle$ $\left\langle\psi_{l, j, k}^{s},\left(g \wedge \chi_{\Omega}\right)^{\vee}\right\rangle$ are absolutely convergent for $f, g \in D$ since $D$ is dense both in $H^{s}\left(R^{d}\right)$ and $H^{-s}\left(R^{d}\right)$. By Lemma 13, (48) can be written as

$$
\begin{aligned}
& \lim _{J \longrightarrow \infty} \int_{R^{d}} \hat{f}(\xi) \hat{\mathcal{G}}(\xi) \chi_{\Omega}(\xi)\left(\widehat{\psi}_{0}(\xi) \widehat{\tilde{\psi}}_{0}(\xi)+\sum_{l=1}^{L} \sum_{j=0}^{J} \widehat{\psi}_{l}\left(M^{*^{-j}} \xi\right) \widehat{\tilde{\psi}}_{l}\left(M^{*^{-j} \xi} \xi\right)\right) d \xi \\
& +\int_{R^{d}} \overline{\bar{g}}(\xi) \chi_{\Omega}(\xi) \sum_{0 \neq k \in Z^{d}}\left(\widehat{f} \chi_{\Omega}\right)(\xi+k)\left(\widehat{\psi}_{0}(\xi) \widehat{\tilde{\psi}}_{0}(\xi+k)+\sum_{l=1}^{L} \sum_{j=0}^{\kappa(k)} \widehat{\psi}_{l}\left(M^{*^{-j}} \xi\right) \overline{\hat{\tilde{\psi}}}_{l}\right. \\
& \left.\times\left(M^{*^{-j}}(\xi+k)\right)^{-}\right) d \xi=\int_{R^{d}} \hat{f}(\xi) \hat{\mathcal{G}}(\xi) \chi_{\Omega}(\xi) d \xi \text { forf }, g \in D .
\end{aligned}
$$

Obviously, (45) and (46) imply (49). Next, we prove the converse implication to complete the proof. Suppose (49) holds. For $0 \neq k \in Z^{d}$, the function

$$
\widehat{\psi}_{0}(\cdot) \widehat{\tilde{\psi}}_{0}(\cdot+k)+\sum_{l=1}^{L} \sum_{j=0}^{k(k)} \widehat{\psi}_{l}\left(M^{*^{-j}} \cdot\right) \widehat{\tilde{\psi}}_{l}\left(M^{*^{-j}}(\cdot+k)\right) \in L^{1}\left(R^{d}\right)
$$

and thus, almost every point in $R^{d}$ is a Lebesgue point of all 
function

$$
\widehat{\psi}_{0}(\cdot) \widehat{\tilde{\psi}}_{0}(\cdot+k)+\sum_{l=1}^{L} \sum_{j=0}^{k(k)} \widehat{\psi}_{l}\left(M^{*^{-j}} \cdot\right) \widehat{\widetilde{\psi}}_{l}\left(M^{*^{-j}}(\cdot+k)\right),
$$

with $0 \neq k \in Z^{d}$. Let $\xi_{0} \in R^{d}$ be such a point. For $0<\varepsilon<1 / 2$ and $0 \neq k_{0} \in Z^{d}$, take $f$ and $g$ such that $\widehat{f}\left(\cdot+k_{0}\right)=\widehat{g}(\cdot)=$ $\chi_{B\left(\xi_{0}, \varepsilon\right.}(\cdot) / \sqrt{\left|B\left(\xi_{0}, \varepsilon\right)\right|}$ in (49), where $B\left(\xi_{0}, \varepsilon\right)=\left\{\xi \in R^{d}: \mid \xi\right.$ $\left.-\xi_{0} \mid<\varepsilon\right\}$. Then, we have

$$
\begin{aligned}
& \frac{1}{\left|B\left(\xi_{0}, \varepsilon\right)\right|} \int_{B\left(\xi_{0}, \varepsilon\right)} X_{\Omega}(\xi) X_{\Omega}\left(\xi+k_{0}\right) \\
& \cdot\left(\widehat{\psi}_{0}(\xi) \overline{\hat{\psi}_{0}\left(\xi+k_{0}\right)}+\sum_{l=1}^{L} \sum_{j=0}^{\kappa\left(k_{0}\right)} \widehat{\psi}_{l}\left(M^{*-1} \xi\right) \overline{\widehat{\psi}^{\sim} l}\left(M^{*-j}\left(\xi+k_{0}\right)\right)\right) d \xi=0,
\end{aligned}
$$

or equivalently,

$$
\begin{aligned}
& \frac{1}{\left|B\left(\xi_{0}, \varepsilon\right)\right|} \int_{B\left(\xi_{0}, \varepsilon\right)} \\
& \cdot\left(\widehat{\psi}_{0}(\xi) \overline{\hat{\psi}_{0}\left(\xi+k_{0}\right)}+\sum_{l=1}^{L} \sum_{j=0}^{k\left(k_{0}\right)} \widehat{\psi}_{l}\left(M^{*-1} \xi\right) \overline{\widehat{\psi}^{\prime} l}\left(M^{*-j}\left(\xi+k_{0}\right)\right)\right) d \xi=0,
\end{aligned}
$$

since

$$
\widehat{\psi}_{0}(\xi) \widehat{\tilde{\psi}}_{0}\left(\xi+k_{0}\right)+\sum_{l=1}^{L} \sum_{j=0}^{\kappa\left(k_{0}\right)} \widehat{\psi}_{l}\left(M^{*^{-j}} \xi\right)
$$

$\widehat{\tilde{\psi}}_{l}\left(M^{*^{-j}}\left(\xi+k_{0}\right)\right)=0$ if $\xi$ and $\xi+k_{0}$ belong to $\Omega$ when $\xi \epsilon$ $B\left(\xi_{0}, \varepsilon\right)$. Letting $\varepsilon \longrightarrow 0$ in (53), we obtain

$$
\widehat{\psi}_{0}\left(\xi_{0}\right) \widehat{\tilde{\psi}}_{0}\left(\xi_{0}+k_{0}\right)+\sum_{l=1}^{L} \sum_{j=0}^{\kappa\left(k_{0}\right)} \widehat{\psi}_{l}\left(M^{*^{-j}} \xi_{0}\right) \widehat{\widetilde{\psi}}_{l}\left(M^{*^{-j}}\left(\xi_{0}+k_{0}\right)\right)=0 .
$$
have

By the arbitrariness of $\xi_{0} \in R^{d}$ and $0 \neq k_{0} \in Z^{d}$, then we $\widehat{\psi}_{0}(\cdot) \widehat{\tilde{\psi}}_{0}(++k)+\sum_{l=1}^{L} \sum_{j=0}^{\kappa(k)} \widehat{\psi}_{l}\left(M^{*-j} \cdot\right) \widehat{\tilde{\psi}}_{l}\left(M^{*-j}(++k)\right)=0 a . e$. on $R^{d}$ for $0 \neq k \in Z^{d}$.

And thus, (46) holds since the above function vanishes out of $\Omega$. Now, we prove (45) holds.

Arbitrarily fix $h \in L^{\infty}(K), \forall$ compact $K \subset \mathbb{R}^{d} \backslash\{0\}$, then there exists a finite set $\left\{k_{1}, k_{2}, \cdots, k_{m}\right\} \subset \mathbb{Z}^{d}$ such that $K=$ $\bigcup_{i=1}^{m}\left[\left([0,1)^{d}+k_{i}\right) \cap K\right]=\bigcup_{i=1}^{m} S_{i}$. Define $h_{i}=h \chi_{S_{i}}$ for $1 \leq i \leq m$. Then, we have $h=\sum_{i=1}^{m} h_{i}$, and thus, (45) holds for $h$ if it holds for each $h_{i}$ with $1 \leq i \leq m$. Next, we prove (45) holds for each $h_{i}$ with $1 \leq i \leq m$. Take $f$ and $g$ in (49) such that

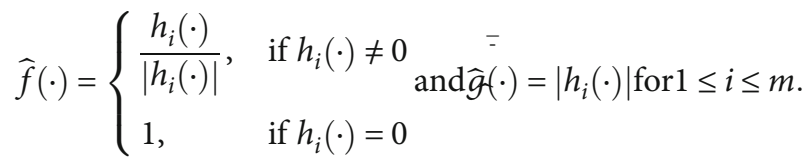

Then, we obtain

$$
\begin{aligned}
& \lim _{J \longrightarrow \infty} \int_{R^{d}} h_{i}(\xi)\left(\widehat{\psi}_{0}(\xi) \widehat{\tilde{\psi}}_{0}(\xi)+\sum_{l=1}^{L} \sum_{j=0}^{J} \widehat{\psi}_{l}\left(M^{*^{-j}} \xi\right) \widehat{\tilde{\psi}}_{l}\left(M^{*^{-j}} \xi\right)\right) d \xi \\
& \quad=\int_{R^{d}} h_{i}(\xi) \chi_{\Omega}(\xi) d \xi .
\end{aligned}
$$

(45) therefore holds for $h_{i}$ and holds for $h$. By the arbitrariness of $h$, we obtain (45). The proof is completed. $\square \square \square$

\section{Constructing Weak Dual Wavelet Frames from a Pair of General Refinable Functions}

This section is devoted to constructing weak dual wavelet frames for $\left(F H^{s}(\Omega), F H^{-s}(\Omega)\right)$ from a pair of general refinable functions under the following assumptions:

Assumption 1. $\psi_{0} \in H^{s}\left(R^{d}\right)$ and $\tilde{\psi}_{0} \in H^{-s}\left(R^{d}\right)$ are $M$-refinable functions with symbols in $L^{\infty}\left(T^{d}\right)$, i.e., there exists $b_{0}$, $\tilde{b}_{0} \in L^{\infty}\left(T^{d}\right)$ such that

$\widehat{\psi}_{0}(\cdot)=b_{0}\left(M^{*-1} \cdot\right) \widehat{\psi}_{0}\left(M^{*-1} \cdot\right)$ and $\widehat{\tilde{\psi}}_{0}(\cdot)=\tilde{b}_{0}\left(M^{*^{-1}} \cdot\right) \widehat{\tilde{\psi}}_{0}\left(M^{*-1} \cdot\right)$ a.e.on $R^{d}$.

Assumption 2. $\left[\widehat{\psi}_{0}, \widehat{\psi}_{0}\right]_{s},\left[\widehat{\tilde{\psi}}_{0}, \widehat{\tilde{\psi}}_{0}\right]_{-s} \in L^{\infty}\left(T^{d}\right)$.

Assumption 3. $\widehat{\psi}_{0} \widehat{\tilde{\psi}}_{0} \chi_{B(0, \delta)} \in L^{\infty}\left(R^{d}\right)$ for some $\delta>0$, where $B(0, \delta)$ denotes the $\delta$-neighborhood of the origin 0 .

Given $L \in N$, let $b_{l}, \tilde{b}_{l} \in L^{\infty}\left(T^{d}\right)$ with $1 \leq l \leq L$ define $\Psi$ $=\left\{\psi_{l}: 1 \leq l \leq L\right\}$ and $\tilde{\Psi}=\left\{\tilde{\psi}_{l}: 1 \leq l \leq L\right\}$ by

$$
\begin{aligned}
& \widehat{\psi}_{l}(\cdot)=b_{l}\left(M^{*^{-1}} \cdot\right) \widehat{\psi}_{0}\left(M^{*^{-1}} \cdot\right), \\
& \widehat{\tilde{\psi}}_{l}(\cdot)=\tilde{b}_{l}\left(M^{*^{-1}} \cdot\right) \widehat{\tilde{\psi}}_{0}\left(M^{*^{-1}} \cdot\right) .
\end{aligned}
$$

Remark 15. By Lemma 10 (i), Assumption 2 is equivalent to the fact that $\left\{T_{k} \psi_{0}: k \in Z^{d}\right\}$ and $\left\{T_{k} \tilde{\psi}_{0}: k \in Z^{d}\right\}$ are Bessel sequences in $H^{s}\left(R^{d}\right)$ and $H^{-s}\left(R^{d}\right)$, respectively. It is easy to check that $\left\{T_{k} \psi_{l}: k \in Z^{d}, 1 \leq l \leq L\right\}$ and $\left\{T_{k} \tilde{\psi}_{l}: k \in Z^{d}, 1 \leq\right.$ $l \leq L\}$ are also Bessel sequences in $H^{s}\left(R^{d}\right)$ and $H^{-s}\left(R^{d}\right)$, respectively. 
Theorem 16. Let $F H^{s}(\Omega)$ and $F H^{-s}(\Omega)$ be reducing subspaces of $H^{s}\left(R^{d}\right)$ and $H^{-s}\left(R^{d}\right)$, respectively, $\psi_{0} \in F H^{s}(\Omega)$ and $\tilde{\psi}_{0} \in F H^{-s}(\Omega)$ two functions satisfy Assumptions 1-3, and let $\Psi=\left\{\psi_{l}: 1 \leq l \leq L\right\}$ and $\tilde{\Psi}=\left\{\tilde{\psi}_{l}: 1 \leq l \leq L\right\}$ be two finite subsets of $F H^{s}(\Omega)$ and $F H^{-s}(\Omega)$, respectively, defined by (59). Assume that there exists a function $\Theta \in L^{\infty}\left(T^{d}\right)$ such that

$\lim _{j \longrightarrow \infty} \int_{\Omega} \Theta\left(M^{*^{-j}} \xi\right) h(\xi) d \xi=\int_{\Omega} h(\xi) d \xi$ forh $\in L^{\infty}(K), \forall$ compact $K \subset R^{d} \backslash\{0\}$

$$
\begin{aligned}
& \Theta\left(M^{*} \cdot\right) b_{0}(\cdot) \tilde{b}_{0}(\cdot+q)+\sum_{l=1}^{L} b_{l}(\cdot) \tilde{b}_{l}(\cdot+q) \\
& =\Theta(\cdot) \delta_{0, q} \text { a.e.on } \sigma\left(\psi_{0}\right) \cap \tau\left(\sigma\left(\tilde{\psi}_{0}\right)-q\right) \text { with } q \in \Gamma_{M^{*}} .
\end{aligned}
$$

Then, $\left(X(\eta, \Psi), X\left(\tilde{\psi}_{0}, \tilde{\Psi}\right)\right)$ is a pair of weak dual wavelet frames for $\left(F H^{s}(\Omega), F H^{-s}(\Omega)\right)$, where $\eta$ is defined by $\widehat{\eta}(\cdot)$ $=\Theta(\cdot) \widehat{\psi}_{0}(\cdot)$.

Proof. First, we claim that the following equation holds:

$$
\begin{aligned}
& \Theta\left(M^{* j} \cdot\right) \widehat{\psi}_{0}\left(M^{*-j} \cdot\right) \widehat{\tilde{\psi}}_{0}\left(M^{*-j}(+n)\right)+\sum_{l=1}^{L} \widehat{\psi}_{l}\left(M^{*-j} \cdot\right) \widehat{\hat{\psi}}_{l}\left(M^{*-j}(\cdot+n)\right) \\
& =\Theta\left(M^{* j-1} \cdot\right) \widehat{\psi}_{0}\left(M^{* j-1} \cdot\right) \widehat{\hat{\psi}}_{0}\left(M^{* j-1}(+n)\right),
\end{aligned}
$$

a.e. on $\mathbb{R}^{d}$ for $n \in \mathbb{Z}^{d}$ and $0 \leq j<\kappa(n)$. Indeed, by Assumption 1 and (59) and the $Z^{d}$-periodicity of $\tilde{b}_{l}$ with 1 $\leq l \leq L$, we have

$$
\begin{aligned}
& \Theta\left(M^{*^{-j}} \cdot\right) \widehat{\psi}_{0}\left(M^{*^{-j}} \cdot\right) \widehat{\hat{\psi}}_{0}\left(M^{*^{-j}}(\cdot+n)\right)+\sum_{l=1}^{L} \widehat{\psi}_{l}\left(M^{*^{-j}} \cdot\right) \widehat{\hat{\psi}}_{l}\left(M^{*^{-j}}(+n)\right) \\
& =\left(\Theta\left(M^{*^{-j}} \cdot\right) b_{0}\left(M^{*^{-j-1}} \cdot\right) \tilde{b}_{0}\left(\overline{M^{* j 1}} \cdot\right)\right. \\
& \left.+\sum_{l=1}^{L} b_{l}\left(M^{* j-1} \cdot\right) \tilde{b}_{l}\left(M^{*^{* 1}} \cdot\right)\right) \widehat{\psi}_{0}\left(M^{* j-1} \cdot\right) \widehat{\tilde{\psi}}_{0}\left(M^{* j-1}(+n)\right) .
\end{aligned}
$$

So (62) holds if

$$
\Theta\left(M^{*-j} \cdot\right) b_{0}\left(M^{* j-1} \cdot\right) \tilde{b}_{0}\left(M^{* j 1} \cdot\right)+\sum_{l=1}^{L} b_{l}\left(M^{*^{j-1}} \cdot\right) \tilde{b}_{l}\left(M^{* *^{\prime 1}} \cdot\right)=\Theta\left(M^{*^{-j-1}} \xi\right)
$$

a.e. on $\mathbb{R}^{d}$ with $\widehat{\psi}_{0}\left(M^{*^{-j-1}} \cdot\right) \widehat{\tilde{\psi}}_{0}\left(M^{*^{-j-1}}(\cdot+n)\right) \neq 0$. Fix $\xi$ with $\widehat{\psi}_{0}\left(M^{*^{-j-1}} \xi\right) \widehat{\tilde{\psi}}_{0}\left(M^{*^{-j-1}}(\xi+n)\right) \neq 0$. Suppose $M^{*^{-j-1}} \xi=\xi$ $'+n_{\xi}$ for some $\xi^{\prime} \in \mathbb{T}^{d}$ and $n_{\xi} \in \mathbb{Z}^{d}$. Then, $\xi^{\prime} \in \sigma\left(\psi_{0}\right) \cap \sigma($ $\tilde{\psi}_{0}$ ) by Lemma 8 , and thus,

$$
\Theta\left(M^{*} \xi^{\prime}\right) b_{0}\left(\xi^{\prime}\right) \tilde{b}_{0}\left(\xi^{\prime}\right)+\sum_{l=1}^{L} b_{l}\left(\xi^{\prime}\right) \tilde{b}_{l}\left(\bar{\xi}^{\prime}\right)=\Theta\left(\xi^{\prime}\right)
$$

by (61). This is equivalent to (64) by the $Z^{d}$-periodicity of $\Theta$, $b_{l}$, and $\tilde{b}_{l}$ with $0 \leq l \leq L$. Therefore, (62) holds.

By Theorem 14, to complete the proof, it is enough to prove that

$$
\begin{aligned}
& \lim _{J \longrightarrow \infty} \int_{R^{d}}\left(\Theta(\xi) \widehat{\psi}_{0}(\xi) \widehat{\hat{\psi}}_{0}(\xi)+\sum_{l=1}^{L} \sum_{j=0}^{J} \widehat{\psi}_{l}\left(M^{*^{-j}} \xi\right) \widehat{\hat{\psi}}_{l}\left(M^{*^{-j}} \xi\right)\right) h(\xi) d \xi \\
& \quad=\int_{R^{d}} \chi_{\Omega}(\xi) h(\xi) d \xi,
\end{aligned}
$$

for $h \in L^{\infty}(K), \forall$ compact $K \subset R^{d} \backslash\{0\}$, and

$$
\begin{aligned}
& \Theta(\cdot) \widehat{\psi}_{0}(\cdot) \widehat{\tilde{\psi}}_{0}(\cdot+n)+\sum_{l=1}^{L} \sum_{j=0}^{\kappa(n)} \widehat{\psi}_{l}\left(M^{*^{-j}} \cdot\right) \widehat{\tilde{\psi}}_{l}\left(M^{*^{-j}}(\cdot+n)\right) \\
& =0 \text { a.e on } \Omega \text { for } 0 \neq n \in \mathbb{Z}^{d} .
\end{aligned}
$$
have

We first prove (67). For $0 \neq n \in Z^{d}$, by using (62), we

$$
\begin{aligned}
\Theta(\cdot) \widehat{\psi}_{0}(\cdot) \widehat{\tilde{\psi}}_{0}(\cdot+n)+\sum_{l=1}^{L} \sum_{j=0}^{\kappa(n)} \widehat{\psi}_{l}\left(M^{*^{-j}} \cdot\right) \widehat{\tilde{\psi}}_{l}\left(M^{*^{-j}}(\cdot+n)\right) \\
=\Theta\left(M^{*^{-1}} \cdot\right) \widehat{\psi}_{0}\left(M^{*^{-1}} \cdot\right) \widehat{\tilde{\psi}}_{0}\left(M^{*^{-1}}(\cdot+n)\right) \\
+\sum_{l=1}^{L} \sum_{j=1}^{\kappa(n)} \widehat{\psi}_{l}\left(M^{*^{-j}} \cdot\right) \widehat{\tilde{\psi}}_{l}\left(M^{*^{-j}}(\cdot+n)\right) \\
=\Theta\left(M^{*^{-2}} \cdot\right) \widehat{\psi}_{0}\left(M^{*^{-2}} \cdot\right) \widehat{\tilde{\psi}}_{0}\left(M^{*^{-2}}(\cdot+n)\right) \\
+\sum_{l=1}^{L} \sum_{j=2}^{\kappa(n)} \widehat{\psi}_{l}\left(M^{*^{-j}} \cdot\right) \widehat{\tilde{\psi}}_{l}\left(M^{*^{-j}}(\cdot+n)\right) \\
=\cdots=\Theta\left(M^{*^{-\kappa(n)}} \cdot\right) \widehat{\psi}_{0}\left(M^{*^{-\kappa(n)}} \cdot\right) \widehat{\tilde{\psi}}_{0}\left(M^{*^{-\kappa(n)}}(\cdot+n)\right) \\
\quad+\sum_{l=1}^{L} \widehat{\psi}_{l}\left(M^{*^{-\kappa(n)}} \cdot\right) \widehat{\tilde{\psi}}_{l}\left(M^{*^{-\kappa(n)}}(\cdot+n)\right) .
\end{aligned}
$$

By Lemma 7, we have $M^{*^{-\kappa(n)-1}} n=n^{\prime}+q$ for some $n^{\prime} \epsilon$ 
$Z^{d}$ and $0 \neq q \in \Gamma_{M^{*}}$. It follows that

$$
\begin{aligned}
& \Theta\left(M^{*^{-\kappa(n)}} \cdot\right) \widehat{\psi}_{0}\left(M^{*^{-\kappa(n)}} \cdot\right) \widehat{\tilde{\psi}}_{0}\left(M^{*^{-\times(n)}}(+n)\right)+\sum_{l=1}^{L} \widehat{\psi}_{l}\left(M^{*^{-\kappa(n)}} \cdot\right) \widehat{\tilde{\psi}}_{l}\left(M^{*^{-K(n)}}(+n)\right)
\end{aligned}
$$

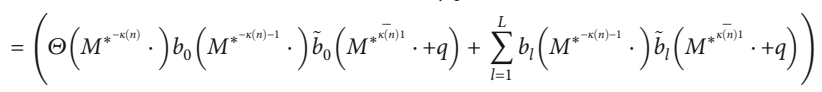

$$
\begin{aligned}
& \times \widehat{\psi}_{0}\left(M^{*^{-x(n)-1}} \cdot\right) \widehat{\tilde{\psi}}_{0}\left(M^{*^{-x(n)-1}}(\cdot+n)\right)=0,
\end{aligned}
$$

by (61), and (67) therefore holds.

Next, we prove (66) holds. By using (62), we have

$$
\begin{aligned}
\Theta(\cdot) \widehat{\psi}_{0}(\cdot) \widehat{\tilde{\psi}}_{0}(\cdot)+\sum_{l=1}^{L} \sum_{j=0}^{J} \widehat{\psi}_{l}\left(M^{*^{-j}} \cdot\right) \widehat{\tilde{\psi}}_{l}\left(M^{*^{-j}} \cdot\right) \\
=\Theta(\cdot) \widehat{\psi}_{0}(\cdot) \widehat{\tilde{\psi}}_{0}(\cdot)+\sum_{l=1}^{L} \widehat{\psi}_{l}(\cdot) \widehat{\tilde{\psi}}_{l}(\cdot)+\sum_{l=1}^{L} \sum_{j=1}^{J} \widehat{\psi}_{l}\left(M^{*^{-j}} \cdot\right) \widehat{\tilde{\psi}}_{l}\left(M^{*^{-j}} \cdot\right) \\
=\Theta\left(M^{*^{-1}} \cdot\right) \widehat{\psi}_{0}\left(M^{*^{-1}} \cdot\right) \widehat{\tilde{\psi}}_{0}\left(M^{*^{-1}} \cdot\right)+\sum_{l=1}^{L} \sum_{j=1}^{J} \widehat{\psi}_{l}\left(M^{*^{-j}} \cdot\right) \widehat{\tilde{\psi}}_{l}\left(M^{*^{-j}} \cdot\right) \\
=\Theta\left(M^{*^{-2}} \cdot\right) \widehat{\psi}_{0}\left(M^{*^{-2}} \cdot\right) \widehat{\tilde{\psi}}_{0}\left(M^{*^{-2}} \cdot\right)+\sum_{l=1}^{L} \sum_{j=2}^{J} \widehat{\psi}_{l}\left(M^{*^{-j}} \cdot\right) \widehat{\tilde{\psi}}_{l}\left(M^{*^{-j}} \cdot\right) \\
=\cdots=\Theta\left(M^{*^{-J}} \cdot\right) \widehat{\psi}_{0}\left(M^{*^{J}} \cdot\right) \widehat{\tilde{\psi}}_{0}\left(M^{*^{-J}} \cdot\right)+\sum_{l=1}^{L} \widehat{\psi}_{l}\left(M^{*^{-J}} \cdot\right) \widehat{\tilde{\psi}}_{l}\left(M^{*^{-J}} \cdot\right) \\
=\left(\Theta\left(M^{*^{-J}} \cdot\right) b_{0}\left(M^{*^{-J-1}} \cdot\right) \tilde{b}_{0}\left(M^{*^{\prime 1}} \cdot\right)+\sum_{l=1}^{L} b_{l}\left(M^{*^{-J-1}} \cdot\right) \tilde{b}_{l}\left(M^{*^{\prime 1}} \cdot\right)\right) \widehat{\psi}_{0} \\
=\left(M^{*^{-J-1}} \cdot\right) \widehat{\tilde{\psi}}_{0}\left(M^{*^{-J-1}} \cdot\right)=\Theta\left(M^{*^{-J-1}} \cdot\right) \widehat{\psi}_{0}\left(M^{*^{-J-1}} \cdot\right) \widehat{\tilde{\psi}}_{0}\left(M^{*^{-J-1}} \cdot\right),
\end{aligned}
$$

where (61) was used in the last equality. And thus,

$$
\begin{gathered}
\lim _{J \longrightarrow \infty} \int_{R^{d}}\left(\Theta(\xi) \widehat{\psi}_{0}(\xi) \widehat{\tilde{\psi}}_{0}(\xi)+\sum_{l=1}^{L} \sum_{j=0}^{J} \widehat{\psi}_{l}\left(M^{*^{-j}} \xi\right) \widehat{\tilde{\psi}}_{l}\left(M^{*^{-j}} \xi\right)\right) h(\xi) d \xi \\
=\lim _{J \longrightarrow \infty} \int_{R^{d}} \Theta\left(M^{*^{-J-1}} \xi\right) \widehat{\psi}_{0}\left(M^{*^{-J-1}} \xi\right) \widehat{\tilde{\psi}}_{0}\left(M^{*^{-J-1}} \xi\right) h(\xi) d \xi \\
=\lim _{J \longrightarrow \infty} \int_{\Omega} \Theta\left(M^{*^{-J-1}} \xi\right) \widehat{\psi}_{0}\left(M^{*^{J-1}} \xi\right) \widehat{\tilde{\psi}}_{0}\left(M^{*^{-J-1}} \xi\right) h(\xi) d \xi
\end{gathered}
$$

for $h \in L^{\infty}(K)$ with compact $K \subset R^{d} \backslash\{0\}$. Since $K$ is compact and $K \subset R^{d} \backslash\{0\}$, then there exists $J_{0}>0$ such that $M^{*^{-J-1}} \xi \in B(0, \delta)$ for $J>J_{0}+1$ and $\xi \in K$. And thus, there exists a constant $M>0$ such that

$$
\left|\Theta\left(M^{*^{-J-1}} \cdot\right) h(\cdot)\left(\widehat{\psi}_{0}\left(M^{*^{-J-1}} \cdot\right) \widehat{\tilde{\psi}}_{0}\left(M^{*^{-J-1}} \cdot\right)-1\right)\right| \leq M|h(\cdot)|,
$$

by Assumption 3. This implies that

$$
\lim _{J \longrightarrow \infty} \int_{\Omega} \Theta\left(M^{*^{-J-1}} \xi\right) h(\xi)\left(\widehat{\psi}_{0}\left(M^{*^{-J-1}} \xi\right) \widehat{\tilde{\psi}}_{0}\left(M^{*^{-J-1}} \xi\right)-1\right) d \xi=0
$$

by Assumptions 1 and 3. Therefore, (66) holds by collecting (71) and (60).

Remark 17.

(i) In the literature, the function $\Theta$ is related to the notion of mixed fundamental function, which plays a significant role in MOEP.

(ii) In the above theorem, (60) is trivial and $\left(X\left(\psi_{0}, \Psi\right)\right.$ ,$\left.X\left(\tilde{\psi}_{0}, \tilde{\Psi}\right)\right)$ is a pair of weak dual wavelet frames for $\left(F H^{s}(\Omega), F H^{-s}(\Omega)\right)$ if $\Theta(\cdot)=1$ a.e. on $T^{d}$

\section{Conclusion}

In this paper, we introduce the notion of weak dual wavelet frames. And for generality, we work under the setting of reducing subspaces of Sobolev spaces, we characterize a pair of weak dual wavelet frames, and by using this characterization, we obtain a mixed oblique extension principle for such weak dual wavelet frames.

\section{Data Availability}

There is no data in our paper.

\section{Conflicts of Interest}

The author declares no conflicts of interest.

\section{Authors' Contributions}

The author read and approved the final manuscript.

\section{Acknowledgments}

This work was supported by the National Natural Science Foundation of China (Grant Nos. 11961072 and 62041212), the Natural Science Basic Research Program of Shanxi (Grant Nos. 2020JM-547 and 2020JM-548), and the Doctoral Research Project of Yan'an University (Grant No. YDBK2017-21).

\section{References}

[1] O. Christensen, An Introduction to Frames and Riesz Bases, Springer, Boston, 2003.

[2] E. Hernández and G. Weiss, A First Course on Wavelets, CRC Press, Boca Raton, 1996.

[3] R. M. Young, An Introduction to Nonharmonic Fourier Series, Academic Press, New York, 1980. 
[4] M. Bownik and Z. Rzeszotnik, "Construction and reconstruction of tight framelets and wavelets via matrix mask functions," Journal of Functional Analysis, vol. 256, pp. 10651105, 2009.

[5] N. Atreas, A. Melas, and T. Stavropoulos, "Affine dual frames and extension principles," Applied and Computational Harmonic Analysis, vol. 36, pp. 51-62, 2014.

[6] M. Bownik, "A characterization of affine dual frames in $\mathrm{L}^{2}\left(\mathrm{R}^{\mathrm{n}}\right)$ ," Applied and Computational Harmonic Analysis, vol. 8, pp. 203-221, 2000.

[7] O. Christensen, H. O. Kim, and R. Y. Kim, "On extensions of wavelet systems to dual pairs of frames," Advances in Computational Mathematics, vol. 42, pp. 489-503, 2016.

[8] O. Christensen, H. Oh Kim, and R. Young Kim, "On Parseval wavelet frames with two or three generators via the unitary extension principle," Canadian Mathematical Bulletin, vol. 57, no. 2, pp. 254-263, 2014.

[9] C. K. Chui and Q. Sun, "Affine frame decompositions and shift-invariant spaces," Applied and Computational Harmonic Analysis, vol. 20, pp. 74-107, 2006.

[10] I. Daubechies, B. Han, A. Ron, and Z. Shen, "Framelets, MRAbased constructions of wavelet frames," Applied and Computational Harmonic Analysis, vol. 14, pp. 1-46, 2003.

[11] I. Daubechies and B. Han, "Pairs of dual wavelet frames from any two refinable functions," Constructive Approximation, vol. 20, no. 3, pp. 325-352, 2004.

[12] M. Ehler, "The multiresolution structure of pairs of dual wavelet frames for a pair of Sobolev spaces," Jaen Journal on Approximation, vol. 2, pp. 193-214, 2010.

[13] B. Han and Z. Shen, "Dual wavelet frames and Riesz bases in Sobolev spaces," Constructive Approximation, vol. 29, pp. 369-406, 2009.

[14] H.-F. Jia and J.-P. Zhang, "A characterization of nonhomogeneous wavelet bi-frames for reducing subspaces of Sobolev spaces," Journal of Inequalities and Applications, vol. 2021, no. $1,2021$.

[15] A. Ron and Z. Shen, "Affine systems in $\mathrm{L}^{2}\left(\mathrm{R}^{\mathrm{d}}\right)$ : the analysis of the analysis operator," Journal of Functional Analysis, vol. 148, pp. 408-447, 1997.

[16] A. Ron and Z. Shen, "Affine systems in $\mathrm{L}^{2}\left(\mathrm{R}^{\mathrm{d}}\right)$ II: dual systems," Journal of Fourier Analysis and applications, vol. 3, pp. 617-637, 1997.

[17] Y.-Z. Li and J.-P. Zhang, "Nonhomogeneous dual wavelet frames and mixed oblique extension principles in Sobolev spaces," Applicable Analysis, vol. 97, no. 7, pp. 1049-1073, 2018.

[18] N. Atreas, M. Papadakis, and T. Stavropoulos, "Extension principles for dual multiwavelet frames of $\mathrm{L}^{2}\left(\mathrm{R}^{s}\right)$ constructed from multirefinable generators," Journal of Fourier Analysis and Applications, vol. 22, no. 4, pp. 854-877, 2016.

[19] C. K. Chui and W. He, "Compactly supported tight frames associated with refinable functions," Applied and Computational Harmonic Analysis, vol. 8, pp. 293-319, 2000.

[20] T. Stavropoulos, "The geometry of extension principles," Houston Journal of Mathematics, vol. 38, pp. 833-853, 2012.

[21] H.-F. Jia and Y.-Z. Li, "Refinable function-based construction of weak (quasi-)affine bi-frames," Journal of Fourier Analysis and Applications, vol. 20, pp. 1145-1170, 2014.
[22] X. Dai, Y. Diao, Q. Gu, and D. Han, "Frame wavelets in subspaces of $\mathrm{L}^{2}\left(\mathrm{R}^{\mathrm{d}}\right)$," Proceedings of the American Mathematical Society, vol. 130, pp. 3259-3267, 2002.

[23] Y.-Z. Li and H.-F. Jia, "Weak nonhomogeneous wavelet biframes for reducing subspaces of Sobolev spaces," Numerical Functional Analysis and Optimization, vol. 38, pp. 181-204, 2017. 